\title{
Making or Rescuing Human Tumour Viruses?
}

BECAUSE the RNA tumour viruses are such notorious disrespecters of species barriers, more than one cautious tumour virologist has voiced the fear that growing an animal cancer virus in human cells may result in the creation of a human cancer virus. And on page 445 of this issue of Nature Aaronson reports experiments which go a long way towards fulfilling this prophecy. Last year in Nature (225, 459; 1970), Aaronson and Todaro reported the not surprising finding that eighteen strains of cultured human fibroblasts, all derived from one person, can be transformed, albeit inefficiently, by mouse sarcoma virus. They also found that the progeny viruses produced by their transformed human cells behaved like perfectly ordinary mouse sarcoma virus. In other words, the passage of the mouse virus in the human cells had not apparently caused any detectable change in the host range of the virus, which still transformed mouse cells far more efficiently than human cells. But on page 445, Aaronson tells quite a different story.

During the past months he has maintained some of the original transformed human cells which he and Todaro isolated last year. These transformed fibroblasts, as previously reported, initially released apparently normal mouse sarcoma and leukaemia virus. (Two viruses are involved because mouse sarcoma virus cannot be isolated free of mouse leukaemia virus, for the latter acts as a helper providing undefined but essential functions which the sarcoma virus genome cannot itself specify.) After sixteen weekly transfers, however, the "mouse" sarcoma virus liberated by these transformed human cells was found to have lost its ability to transform Swiss mouse cells but it now transformed human cells much more efficiently than before. Indeed, after such prolonged passage in human cells, the "mouse" sarcoma virus transformed human cells almost as efficiently as it transformed rat cells whereas before passage in human cells the virus transformed rat cells far more efficiently than human cells.

Clearly the host range of the virus had changed markedly as a result of its prolonged stay in human cells. This fact alone, of course, suggests that the changed host range results from some genetic change, rather than a phenotypic change, for example in the virus coat, which might be expected to be immediately apparent. This possibility has to be rigorously proven, however, for it has some startling implications. And so Aaronson passaged for three weeks the virus produced by the transformed human cells in rat cells, arguing that if the changed host range was phenotypic rather than genetic this passage might well eliminate it. But in fact after three weeks in rat cells the virus was still unable to transform mouse cells but transformed human cells even more efficiently. Aaronson next established that both the sarcoma and leukaemia viruses emerging from transformed human cells, after prolonged passage, had identically changed host ranges; both had apparently suffered the same genetic change. He then showed that the same type of sarcoma virus being helped by a different strain of mouse leukaemia virus suffered the same change after passage in human cells.

The two sorts of leukaemia virus helpers used have distinct antigens so that they, and the sarcoma viruses they help, can be differentiated by immunochemical tests. Antisera directed against one sarcoma/leukaemia virus complex do not neutralize the other. As judged by this test the sarcoma/leukaemia viruses emerging from transformed human cells retain at least some of their murine antigens. The viruses adapted to human cells are still specifically inactivated by antisera against the corresponding parental stocks of mouse virus. On the other hand antisera against either type of adapted sarcoma/leukaemia virus complex inactivated both adapted virus complexes but had no detectable effect on either parental virus stock. The prolonged passage of different types of murine sarcoma/leukaemia virus complexes in human cells seems to result not only in a similar change in host range but also in the acquisition of common surface antigens that cannot be detected in the original stocks.

Accepting for the moment that these changes are genetic, how might they arise? There are two obvious possibilities; multiplication and selection of mutants arising during the passage in human cells or recombination between human cell and mouse viral genomes, and Aaronson plumps for the second. All his results certainly suggest that the change in host range of these adapted viruses is genetically stable and, because two antigenically distinct types of sarcoma/leukaemia virus complex are identically changed, it seems perhaps unlikely that this change would arise from the selection of random mutations in independently growing populations. Furthermore, of course, the discovery of reverse transcriptase provides the enzymatic machinery necessary for recombination between the genome of a tumour virus and its host. There is now little doubt that transformation by the RNA tumour viruses involves the synthesis of a double stranded DNA copy of the transforming viral genome, and the integration of this viral DNA into one or more host chromosomes. Although the mechanism of this process remains obscure it seems likely that it involves breakage and rejoining of DNA molecules, events which probably also give rise to recombination. One does not therefore have to stretch the imagination far to picture recombination occurring between a tumour virus genome and that of its host.

What sort of genes are likely to be involved in this process? As Aaronson speculates, the recombination might be between the mouse sarcoma virus genomes and those of putative, latent human viruses, integrated into human chromosomes. If this were the case the new antigens on the surface of recombinant, adapted mouse sarcoma virus might be specified by genes of the putative, latent human virus. In other words these antigens might provide markers for human cancer viruses. Needless to say, all this is for the moment pure, and some may say unwarranted, speculation. But it should not be all that difficult to put it to the acid test of experiment and in any event the changes in mouse sarcoma virus after prolonged passage in human cells raise some fascinating questions about the stability and determination of the host range of animal tumour viruses. Aaronson may not have rescued human tumour virus genes but he has gone some way along the road to making such a virus. 